\title{
Central Limit Theorem for Additive Functionals of Reversible Markov Processes and Applications to Simple Exclusions
}

\author{
C. Kipnis ${ }^{1}$ and S. R. S. Varadhan ${ }^{2 *}$ \\ 1 Centre de Mathematiques Appliqueés, Ecole Polytechnique, F-91128 Palaiseau, France \\ 2 Courant Institute, New York University, New York 10012, USA
}

\begin{abstract}
We prove a functional central limit theorem for additive functionals of stationary reversible ergodic Markov chains under virtually no assumptions other than the necessary ones. We use these results to study the asymptotic behavior of a tagged particle in an infinite particle system performing simple excluded random walk.
\end{abstract}

\section{Introduction}

In a recent work Lebowitz and Spohn [4] proved that diffusion of color for "mechanically" identical particles with two colors as well as convergence to local equilibrium in the hydrodynamical limit were related to the asymptotics, after proper rescaling, of the movements of one or more tagged particles of the system.

Since for the moment purely mechanical systems seem to be out of reach, several models which are to lesser or greater extent stochastic have been proposed. In [3] Kipnis et al. considered the case of a one dimensional system of hard rods with stochastic collisions. In this paper we consider the case of the so-called simple exclusion process.

The intuitive description of the process is the following: Infinitely many particles move on $\mathbb{Z}^{d}$ according to a simple random walk with exponential (mean one) holding time at each site and jump law $p(x)$ which is symmetric, i.e. satisfies $p(x)=p(-x)$ for all $x$ 's. However if a particle attempts a transition to a site already occupied, the jump is suppressed.

The key remark is that, due to invariance of the mechanics under translations, the evolution of the rest of the medium seen from an observer sitting on a tagged particle follows a Markovian evolution (which possesses many reversible probabilities) and that the movement of the tagged particle (the observer) in the absolute frame is in a certain sense "driven" by this Markov process.

* Supported by NSF Grant MCS-8301364, ONR Contract N00014-81-K-0012 and a Fellowship from John S. Guggenheim Memorial Foundation 
In Sect. 1 we prove an abstract theorem on weak convergence to Brownian motion of a process driven by a reversible Markov process.

In Sect. 2 we prove that our tagged particle satisfies the conditions described in Sect. 1 to conclude that if $x_{t}$ denotes the position of the tagged particle then $\varepsilon x_{t \varepsilon}-2$ converges to a Brownian motion with finite diffusion constant $D$. (Notice that before taking the limit the random process $x_{t}$ is not Markovian due to the interactions!)

However it has been shown by Arratia [1] that when $d=1$ and $p(1)=p(-1)=$ $1 / 2$, the diffusion constant $D$ is equal to zero and the correct scaling needed to obtain a nontrivial limit is $\varepsilon x_{t \varepsilon}-4$. We therefore prove that if $d \geqq 2$ or $d=1$ and $p(x)$ is concentrated on more than two points then $D$ is strictly positive.

In Sect. 3. we prove that two different tagged particles become independent in the limit contrary to what happens in [3] where they remain correlated.

In Sect. 4. we apply our theorem to a jump process in random environment obtained by first choosing (randomly) rates for each bond of nearest-neighbor points of the lattice $\mathbb{Z}^{d}$ and letting the particle jump across a bond with the aforementioned rate.

\section{Convergence to Brownian Motion of an Arbitrary Reversible Velocity Process}

The question of proving a central limit theorem for the partial sums

$$
X_{n}=\sum_{j=1}^{n} Z_{j}
$$

of a sequence $Z_{1}, Z_{2}, \ldots, Z_{n}, \ldots$ of random variables has been studied exhaustively in probability theory for at least fifty years. We will limit our discussion here to the situation where $\left\{Z_{j}\right\}$ forms a stationary sequence in the strict sense. If the $Z_{j}$ 's are independent with mean zero and variance $\sigma^{2}$ then the classical central limit theorem asserts that the distribution of $X_{n} / \sqrt{ } n$ converges to a normal distribution with mean 0 and variance $\sigma^{2}$. The invariance principle asserts that the distribution of the stochastic process $X_{n}(t)=1 / \sqrt{ } n X_{[n t]}$ converges weakly in the Skorohod space to the distribution of a Brownian motion with variance $\sigma^{2}$.

These results have been generalized to the situation where the independence of the random variables $\left\{Z_{j}\right\}$ is replaced by asymptotic independence of one type or other. The assumptions are often difficult to check in specific circumstances.

One special situation where analogs of the classical results are valid without additional assumptions is the following:

Theorem 1.1. Let $\left\{Z_{j}:-\infty<j<\infty\right\}$ be a stationary ergodic process such that $E\left[Z_{n+1} \mid F_{n}\right]=0$ a.e., where $F_{n}$ is the $\sigma$-field generated by $Z_{j}$ for $j \leqq n$. For such $a$ martingale difference sequence the distribution of $X_{n}(t)=1 / \sqrt{ } n\left[Z_{1}+\cdots+Z_{[n t]}\right]$ converges weakly to the distribution of Brownian motion with variance $\sigma^{2}$ provided $E Z_{n}^{2}=\sigma^{2}<\infty$.

Proof. The above theorem is deduced easily from Theorem 3.2 of [2] where condition (b) can be checked easily in our situation.

Remark 1.2. If $\left\{Z_{j}\right\}$ is a vector valued sequence one has an identical theorem where 
one obtains in the limit a multidimensional Brownian motion with a covariance matrix identical to that of any $Z_{j}$.

Suppose $\left\{y_{j}\right\},-\infty<j<\infty$ is a stationary Markov chain on a state space $X$ and $Z_{j}=V\left(y_{j}\right)$. Let us suppose that $\pi(d x)$ is the common invariant distribution and $q(x, d y)$ is the transition probability of the chain. Let us assume that the chain is ergodic. We denote by the symbol $q$ the operator $(q f)(x)=\int f(y) q(x, d y)$ as well. Suppose $V(x)=f(x)-(q f)(x)$ for some bounded function $f(x)$. Then $V(x)$ has necessarily mean 0 with respect to $\pi$ and it is natural to ask if the central limit theorem is valid for

$$
X_{n}=\sum_{j=1}^{n} V\left(y_{j}\right)
$$

If we define

$$
Z_{j}^{\prime}=f\left(y_{j}\right)-(q f)\left(y_{j-1}\right)
$$

then an elementary computation shows that

$$
E\left[Z_{n+1}^{\prime} \mid F_{n}\right]=E\left[f\left(y_{n+1}\right) \mid F_{n}\right]-(q f)\left(y_{n}\right)=(q f)\left(y_{n}\right)-(q f)\left(y_{n}\right)=0 .
$$

Moreover if

$$
X_{n}^{\prime}=\sum_{j=1}^{n} Z_{j}^{\prime}
$$

then

$$
X_{n}=\sum_{j=1}^{n} f\left(y_{j}\right)-\sum_{j=1}^{n}(q f)\left(y_{j}\right)=\sum_{j=1}^{n} Z_{j}^{\prime}+(q f)\left(y_{0}\right)-(q f)\left(y_{n}\right) .
$$

We write $X_{n}=X_{n}^{\prime}+X_{n}^{\prime \prime}$, where $X_{n}^{\prime \prime}=(q f)\left(y_{0}\right)-(q f)\left(y_{n}\right)$. Since $X_{n}^{\prime \prime} / \sqrt{n}$ is negligible as $n \rightarrow \infty$, the central limit theorem for $X_{n}$ follows from the central limit theorem for $X_{n}^{\prime}$ which is an application of Theorem 1.1.

Although the approach is quite elementary, when the underlying Markov chain $\left\{y_{j}\right\}$ is reversible the method is powerful enough to establish the central limit theorem and the invariance principle for the most general $V$ that one can expect the theory to hold.

Let $q(x, d y)$ be a translation probability on a state space $X$ with $\pi(d x)$ as a reversible stationary probability. We will assume that the stationary Markov process $P$ with $\pi$ as marginal distribution and $q(x, d y)$ with transition probability is ergodic. Let $V(y)$ be a function on $X$ with $\int V^{2}(x) \pi(d x)<\infty$, and we will assume that $\int V(x) \pi(d x)=0$ and

$$
\lim _{n \rightarrow \infty} \frac{1}{n} E^{P}\left[\left(V\left(y_{1}\right)+\cdots+V\left(y_{n}\right)\right)^{2}\right]=\sigma^{2}<\infty .
$$

If we denote by $e_{V}(d \lambda)$, the spectral measure of $V$ corresponding to the selfadjoint operator $q$ on $L_{2}(X, \pi)$, we obtain

$$
\sigma^{2}=\int \frac{1+\lambda}{1-\lambda} e_{V}(d \lambda)<\infty .
$$


The condition that $\sigma^{2}<\infty$ is therefore equivalent to the condition $\int 1 / 1-\lambda e_{V}(d \lambda)<$ $\infty$ or $V \in \operatorname{Range}(I-q)^{1 / 2}$. This can be verified by checking the inequality

$$
\left|\int V(y) \phi(y) \pi(d y)\right| \leqq C\left(\int((I-q) \phi)(y) \phi(y) \pi(d y)\right)^{1 / 2}
$$

for all test functions $\phi$ in $L_{2}(X, \pi)$. If we introduce the inner products

$$
\begin{aligned}
\left\langle f_{1}, f_{2}\right\rangle & =\int f_{1}(y) f_{2}(y) \pi(d y), \\
\left\langle f_{1}, f_{2}\right\rangle_{1} & =\int\left((I-q) f_{1}\right)(y) f_{2}(y) \pi(d y),
\end{aligned}
$$

then our assumption can be restated as $\langle V, V\rangle_{-1}\left\langle\infty\right.$, where \langle\rangle$_{-1}$ is dual to \langle\rangle$_{1}$ under the pairing \langle\rangle .

We denote by $\Omega$ the space of all $X$-valued sequences $\left\{y_{j}\right\},-\infty<j<\infty$ and by $F_{n}$ the $\sigma$-field generated by $y_{j}$ for $j \leqq n$. We have the measure $P$ on $(\Omega, F)$, where $F=V_{n} F_{n}$.

Theorem 1.3. Let $V$ in $L_{2}(x, \pi)$ satisfy condition (1.1). Then the sequence

$$
X_{n}=\sum_{j=1}^{n} V\left(y_{j}\right)
$$

can be written as $X_{n}=M_{n}+\xi_{n}$, where $M_{n}$ is a martingale relative to $\left(\Omega, F_{n}, P\right)$ and

$$
\lim _{n \rightarrow \infty} \frac{1}{\sqrt{ } n} \sup _{1 \leqq j \leqq n}\left|\xi_{j}\right|=0 \text { in probability }(P) \text {. }
$$

Moreover

$$
\lim _{n \rightarrow \infty} \frac{1}{n} E^{P}\left|\xi_{n}\right|^{2}=0
$$

Proof. We denote by $u_{\varepsilon}$ the solution of the equation

$$
(1+\varepsilon) u_{\varepsilon}-q u_{\varepsilon}=V
$$

for $\varepsilon>0$. We will investigate the behavior of $u_{\varepsilon}$ as $\varepsilon \rightarrow 0$. We can rewrite (1.5) as

$$
u_{\varepsilon}-q u_{\varepsilon}=V-\varepsilon u_{\varepsilon} \text {. }
$$

Therefore for every $n \geqq 0$,

$$
E^{P}\left\{u_{\varepsilon}\left(y_{n+1}\right)-u_{\varepsilon}\left(y_{n}\right)-\varepsilon u_{\varepsilon}\left(y_{n}\right)+V\left(y_{n}\right) \mid F_{n}\right\}=0 .
$$

If we define

$$
M_{n}^{\varepsilon}=\sum_{j=0}^{n-1}\left[u_{\varepsilon}\left(y_{j+1}\right)-u_{\varepsilon}\left(y_{j}\right)+V_{\varepsilon}\left(y_{j}\right)\right],
$$

where $V_{\varepsilon}=V-\varepsilon u_{\varepsilon}$, then for each $\varepsilon>0, M_{n}^{\varepsilon}$ if a martingale relative to $\left(\Omega, F_{n}, P\right)$. We can now express for each $\varepsilon>0, X_{n}=M_{n}^{\varepsilon}+\xi_{n}^{\varepsilon}+\eta_{n}^{\varepsilon}$, where

$$
\xi_{n}^{\varepsilon}=-\sum_{j=0}^{n-1}\left[u_{\varepsilon}\left(y_{j+1}\right)-u_{\varepsilon}\left(y_{j}\right)\right]
$$


and

$$
\eta_{n}^{\varepsilon}=\sum_{j=0}^{n-1}\left(\varepsilon u_{\varepsilon}\right)\left(y_{j}\right)
$$

We shall now establish that for each $n \geqq 1$,

$$
\begin{aligned}
& \lim _{\varepsilon \rightarrow 0} M_{n}^{\varepsilon}=M_{n} \text { exists in } L_{2}(\Omega, P), \\
& \lim _{\varepsilon \rightarrow 0} \xi_{n}^{\varepsilon}=\xi_{n} \text { exists in } L_{2}(\Omega, P), \\
& \lim _{\varepsilon \rightarrow 0} \eta_{n}^{\varepsilon}=0 \text { in } L_{2}(\Omega, P) .
\end{aligned}
$$

It follows then that $X_{n}=M_{n}+\xi_{n}$. We will then establish (1.3) and (1.4) for the sequence $\left\{\xi_{n}\right\}$. Since $M_{n}^{\varepsilon}$ is a martingale with stationary increments in order to show that $M_{n}^{\varepsilon}$ has a limit in $L_{2}(\Omega, P)$, it is sufficient to check that

$$
\lim _{\varepsilon \rightarrow 0}\left\{u_{\varepsilon}\left(y_{2}\right)-u_{\varepsilon}\left(y_{1}\right)+V_{\varepsilon}\left(y_{1}\right\} \text { exists in } L_{2}(\Omega, P) .\right.
$$

From relation (1.6) the above limit is of the form

$$
\lim _{\varepsilon \rightarrow 0}\left\{u_{\varepsilon}\left(y_{2}\right)-\left(q u_{\varepsilon}\right)\left(y_{1}\right)\right\} .
$$

An easy computation yields

$$
E^{P}\left[f\left(y_{2}\right)-(q f)\left(y_{1}\right)\right]^{2}=\left\langle f,\left(I-q^{2}\right) f\right\rangle .
$$

Therefore we need to check only that

$$
\lim _{\substack{\varepsilon_{1} \rightarrow 0 \\ \varepsilon_{2} \rightarrow 0}}\left\langle u_{\varepsilon_{1}}-u_{\varepsilon_{2}},\left(I-q^{2}\right)\left(u_{\varepsilon_{1}}-u_{\varepsilon_{2}}\right)\right\rangle=0 .
$$

Equation (1.11) can be calculated in terms of spectral measure $e_{V}(d \lambda)$ and the formula reads

$$
\begin{aligned}
\left\langle u_{\varepsilon_{1}}-u_{\varepsilon_{2}},\left(I-q^{2}\right)\left(u_{\varepsilon_{1}}-u_{\varepsilon_{2}}\right)\right\rangle & =\int_{-1}^{1}\left(1-\lambda^{2}\right)\left(\frac{1}{1+\varepsilon_{1}-\lambda}-\frac{1}{1+\varepsilon_{2}-\lambda}\right)^{2} e_{V}(d \lambda) \\
& =\int_{-1}^{1} \frac{\left(\varepsilon_{2}-\varepsilon_{1}\right)^{2}\left(1-\lambda^{2}\right)}{\left(1+\varepsilon_{1}-\lambda\right)^{2}\left(1+\varepsilon_{2}-\lambda\right)^{2}} e_{V}(d \lambda) .
\end{aligned}
$$

If we assume that $\varepsilon_{2} \geqq \varepsilon_{1}>0$ the integrand is dominated by $\left(\varepsilon_{2}^{2}\left(1-\lambda^{2}\right) /(1-\lambda)^{2} \varepsilon_{2}^{2}\right)=$ $(1+\lambda) /(1-\lambda)$. Since our basic hypothesis is that $\int(1+\lambda) /(1-\lambda) e_{V}(d \lambda)<\infty$, we have (1.7) by the dominated convergence theorem. By a similar argument we also obtain

$$
\lim _{\substack{\varepsilon_{1} \rightarrow 0 \\ \varepsilon_{2} \rightarrow 0}}\left\langle u_{\varepsilon_{1}}-u_{\varepsilon_{2}},(I-q)\left(u_{\varepsilon_{1}}-u_{\varepsilon_{2}}\right)\right\rangle=0
$$


A similar calculation also yields

$$
\lim _{\varepsilon \rightarrow 0}\left\langle u_{\varepsilon},\left(I-q^{2}\right) u_{\varepsilon}\right\rangle=\int \frac{1+\lambda}{1-\lambda} e_{V}(d \lambda)=\sigma^{2} .
$$

Equation (1.9) involves proving

$$
\lim _{\varepsilon \rightarrow 0} \varepsilon\left\|u_{\varepsilon}\right\|=0 .
$$

We will actually prove the stronger version,

$$
\lim _{\varepsilon \rightarrow 0} \varepsilon\left\langle u_{\varepsilon}, u_{\varepsilon}\right\rangle=0
$$

Again by spectral calculation

$$
\varepsilon\left\langle u_{\varepsilon}, u_{\varepsilon}\right\rangle=\int_{-1}^{1} \frac{\varepsilon}{(1+\varepsilon-\lambda)^{2}} e_{V}(d \lambda) .
$$

The integrand goes to zero and is dominated by $1 /(1-\lambda)$, which is integrable with respect to $e_{V}(d \lambda)$. Since $X_{n}=M_{n}^{\varepsilon}+\xi_{n}^{\varepsilon}+\eta_{n}^{\varepsilon}$ and is independent of $\varepsilon>0,(1.7)$ and (1.9) clearly imply (1.8).

We now concentrate on proving (1.3) and (1.4) for the sequence $\left\{\xi_{n}\right\}$. We first prove (1.4). Clearly for every $\varepsilon>0$,

$$
\xi_{n}=M_{n}^{\varepsilon}-M_{n}+\xi_{n}^{\varepsilon}+\eta_{n}^{\varepsilon} .
$$

Since $M_{n}^{\varepsilon}-M_{n}$ is a martingale with stationary increments,

$$
\begin{aligned}
\frac{1}{n} E^{P}\left|\xi_{n}\right|^{2} & \leqq \frac{3}{n} E^{P}\left|M_{n}^{\varepsilon}-M_{n}\right|^{2}+\frac{3}{n} E^{P}\left|\xi_{n}^{\varepsilon}\right|^{2}+\frac{3}{n} E^{P}\left|\eta_{n}^{\varepsilon}\right|^{2} \\
& =3 E^{P}\left|M_{1}^{\varepsilon}-M_{1}\right|^{2}+\frac{3}{n} E^{P}\left|\xi_{n}^{\varepsilon}\right|^{2}+\frac{3}{n} E^{P}\left|\eta_{n}^{\varepsilon}\right|^{2}
\end{aligned}
$$

We have

$$
\lim _{\varepsilon \rightarrow 0} E^{P}\left|M_{1}^{\varepsilon}-M_{1}\right|^{2}=0 .
$$

Therefore it is enough to choose $\varepsilon=1 / n$ and then show that

$$
\lim _{n \rightarrow \infty} \frac{1}{n} E^{P}\left|\xi_{n}^{1 / n}\right|^{2}=0 \text { and } \quad \lim _{n \rightarrow \infty} \frac{1}{n} E^{P}\left|\eta_{n}^{1 / n}\right|^{2}=0
$$

Clearly

$$
\begin{aligned}
E^{P}\left|\xi_{n}^{1 / n}\right|^{2} & =E^{P}\left|u_{1 / n}\left(y_{n}\right)-u_{1 / n}\left(y_{0}\right)\right|^{2} \leqq 4 E^{P}\left|u_{1 / n}\left(y_{0}\right)\right|^{2} \\
& =4\left\langle u_{1 / n}, u_{1 / n}\right\rangle=o(n) \quad \text { by }(1.12) .
\end{aligned}
$$

Moreover

$$
\begin{aligned}
E^{P}\left|\eta_{n}^{1 / n}\right|^{2} & =E^{P}\left[\frac{1}{n} \sum_{j=0}^{n-1} u_{1 / n}\left(y_{j}\right)\right]^{2} \leqq E^{P}\left|u_{1 / n}\left(y_{0}\right)\right|^{2} \\
& =\left\langle u_{1 / n}, u_{1 / n}\right\rangle=o(n) \text { again by }(1.12)
\end{aligned}
$$


Now we turn to (1.3). We need Lemma 1.4 which we will use but prove after completing the argument;

$$
\sup _{1 \leqq j \leqq n}\left|\xi_{j}\right| \leqq \sup _{1 \leqq j \leqq n}\left|M_{j}^{1 / n}-M_{j}\right|+\sup _{1 \leqq j \leqq n}\left|\xi_{j}^{1 / n}\right|+\sup _{1 \leqq j \leqq n}\left|\eta_{j}^{1 / n}\right| .
$$

Now by Doob's inequality

$$
\begin{aligned}
P\left[\sup _{1 \leqq j \leqq n}\left|M_{j}^{1 / n}-M_{j}\right| \geqq \delta \sqrt{n}\right] & \leqq \frac{1}{n \delta^{2}} E^{P}\left[M_{n}^{1 / n}-M_{n}\right]^{2} \\
& =\frac{1}{\delta^{2}} E^{P}\left[M_{1}^{1 / n}-M_{1}\right]^{2}=o(1) \text { as } n \rightarrow \infty \\
P\left[\sup _{1 \leqq j \leqq n}\left|\eta_{j}^{1 / n}\right| \geqq \delta \sqrt{n}\right] & \leqq P\left[\frac{1}{n} \sum_{0}^{n-1}\left|u_{1 / n}\left(y_{j}\right)\right| \geqq \delta \sqrt{n}\right] \\
& \leqq \frac{1}{n \delta^{2}} E^{P}\left[\frac{1}{n} \sum_{0}^{n-1}\left|u_{1 / n}\left(y_{j}\right)\right|\right]^{2} \leqq \frac{1}{n \delta^{2}}\left\langle u_{1 / n}, u_{1 / n}\right\rangle \\
& =o(1) \text { as } n \rightarrow \infty
\end{aligned}
$$

Finally

$$
\begin{aligned}
& \sup _{1 \leqq j \leqq n}\left|\xi_{j}^{1 / n}\right|=\sup _{1 \leqq j \leqq n}\left|u_{1 / n}\left(y_{j}\right)-u_{1 / n}\left(y_{0}\right)\right| \leqq \sup _{1 \leqq j \leqq n}\left|u_{1 / n}\left(y_{j}\right)\right|+\left|u_{1 / n}\left(y_{0}\right)\right|, \\
& \begin{aligned}
P\left[\left|u_{1 / n}\left(y_{0}\right)\right| \geqq \delta \sqrt{n}\right] & \leqq \frac{1}{n \delta^{2}} E^{P}\left[u_{1 / n}\left(y_{0}\right)\right]^{2}=\frac{1}{n \delta^{2}}\left\langle u_{1 / n}, u_{1 / n}\right\rangle \\
& =o(1) \text { as } n \rightarrow \infty .
\end{aligned}
\end{aligned}
$$

As for $\sup \left|u_{1 / n}\left(y_{j}\right)\right|$, we write

$1 \leqq j \leqq n$

$$
\sup _{1 \leqq j \leqq n}\left|u_{1 / n}\left(y_{j}\right)\right| \leqq \sup _{1 \leqq j \leqq n}\left|u_{\varepsilon}\left(y_{j}\right)-u_{1 / n}\left(y_{j}\right)\right|+\sup _{1 \leqq j \leqq n}\left|u_{\varepsilon}\left(y_{j}\right)\right|
$$

for some $\varepsilon>0$.

$$
P\left[\sup _{1 \leqq j \leqq n}\left|u_{\varepsilon}\left(y_{j}\right)\right| \geqq \delta \sqrt{n}\right] \leqq n P\left[\left|u_{\varepsilon}\left(y_{0}\right)\right| \geqq \delta \sqrt{n}\right]=o(1),
$$

because $E^{P}\left|u_{\varepsilon}\left(y_{0}\right)\right|^{2}<\infty$.

We use Lemma 1.4 to estimate

$$
\begin{aligned}
& P\left[\sup \left|u_{\varepsilon}\left(y_{j}\right)-u_{1 / n}\left(y_{j}\right)\right| \geqq \delta \sqrt{n}\right] \\
& \quad \leqq \frac{3}{\delta \sqrt{ } n}\left(n\left\langle u_{\varepsilon}-u_{1 / n},(I-q)\left(u_{\varepsilon}-u_{1 / n}\right)\right\rangle+\left\langle u_{\varepsilon}-u_{1 / n}, u_{\varepsilon}-u_{1 / n}\right\rangle\right)^{1 / 2},
\end{aligned}
$$

letting $n \rightarrow \infty$,

$$
\begin{aligned}
& \limsup _{n \rightarrow \infty} P\left[\sup _{1 \leqq j \leqq n}\left|u_{\varepsilon}\left(y_{j}\right)-u_{1 / n}\left(y_{j}\right)\right| \geqq \delta \sqrt{n}\right] \\
& \quad \leqq \frac{3}{\delta_{n \rightarrow \infty}} \lim _{n \rightarrow \infty}\left\langle u_{\varepsilon}-u_{1 / n},(I-q)\left(u_{\varepsilon}-u_{1 / n}\right)\right\rangle^{1 / 2}
\end{aligned}
$$


If we let $\varepsilon \rightarrow 0$, we obtain

$$
\begin{aligned}
& \lim _{\varepsilon \rightarrow 0} \limsup _{n \rightarrow \infty} P\left[\sup _{1 \leqq j \leqq n}\left|u_{\varepsilon}\left(y_{j}\right)-u_{1 / n}\left(y_{j}\right)\right| \geqq \delta \sqrt{n}\right] \\
& \quad \leqq \frac{3}{\delta_{\varepsilon \rightarrow 0}} \lim _{n \rightarrow \infty}\left\langle u_{\varepsilon}-u_{1 / n},(I-q)\left(u_{\varepsilon}-u_{1 / n}\right)\right\rangle \\
& \quad=0 \text { by }(1.1 \mathrm{a}),
\end{aligned}
$$

and we are done.

Lemma 1.4. Let $f(x)$ be an arbitrary function in $L_{2}(X, \pi)$. Let

$$
\langle f, f\rangle=a,\langle f,(I-q) f\rangle=b .
$$

Then

$$
P\left\{\sup _{0 \leqq j \leqq n}\left|f\left(y_{j}\right)\right| \geqq l\right\} \leqq \frac{3}{l} \sqrt{a+n b} .
$$

Proof. Let $G=\{x:|f(x)| \geqq l\}$ and let $\tau$ be the hitting time of $G$, i.e. $\tau=\left\{\inf j: y_{j} \in G\right\}$. Then

$$
P\left\{\sup _{0 \leqq j \leqq n}\left|f\left(y_{j}\right)\right| \geqq l\right\}=P\{\tau \leqq n\} .
$$

Let us consider the function $\phi_{\rho}(x)=E^{P_{x}}\left\{\rho^{\tau}\right\}$, where $P_{x}$ is the Markov measure on the space of sequences $\left\{y_{j}\right\}$ for $j \geqq 0$ initialized to start from $x$ at time 0 . Then

$$
P\{\tau \leqq y\} \leqq \rho^{-n} \int \phi_{\rho}(x) \pi(d x) \leqq \rho^{-n}\left[\int \phi_{\rho}^{2}(x) \pi(d x)\right]^{1 / 2} .
$$

To estimate the last term we note that

$$
\phi_{\rho}=\rho q \phi_{\rho} \text { on } G^{c}, \quad \phi_{\rho}=1 \text { on } G .
$$

By the Dirichlet principle

$$
\int \phi_{\rho}^{2}(x) d \pi \leqq\left\langle\phi_{\rho}, \phi_{\rho}\right\rangle+\frac{\rho}{1-\rho}\left\langle\phi_{\rho},(I-q) \phi_{\phi}\right\rangle \leqq\langle\omega, \omega\rangle+\frac{\rho}{1-\rho}\langle\omega,(I-q) \omega\rangle
$$

for any $\omega$ which is 1 on $G$. The function $(|f(x)| \Lambda l / l)=\bar{\omega}$ is an admissible choice;

$$
\langle\bar{\omega}, \bar{\omega}\rangle \leqq \frac{1}{l^{2}}\langle f, f\rangle=\frac{a}{l^{2}}, \quad\langle\bar{\omega},(I-q) \bar{\omega}\rangle \leqq \frac{1}{l^{2}}\langle f,(I-q) f\rangle=\frac{b}{l^{2}} .
$$

Therefore

$$
P[\tau \leqq n] \leqq \rho^{-n}\left(\frac{a}{l^{2}}+\frac{\rho}{1-\rho} \frac{b}{l^{2}}\right)^{1 / 2} .
$$

Taking $\rho=e^{-1 / n}$ we obtain (1.13).

Corollary 1.5. If $V$ satisfies (1.1), then the distribution of $X_{n}(t)=1 / \sqrt{ } n \quad X_{[n t]}=$ $(1 / \sqrt{n}) \sum_{j=1}^{[n t]} V\left(y_{j}\right)$ under $P$ converges to Brownian motion with variance $\sigma^{2}$ weakly in the Skorohod space on any finite interval. Moreover $\sigma^{2}$ is given by (1.1). 
Remark 1.6. If $V$ is vector valued then essentially the same result is valid. The vector valued process $X_{n}(t)$ converges weakly to a multidimensional Brownian motion with the corresponding covariance matrix.

Remark 1.7. If we replace $P$ by $P_{x}$ the Markov measure starting from $x$ at time 0 , then the distribution of $X_{n}(t)$ under $P_{x}$ will again converge to the correct Brownian motion but the convergence is in measure as functions of $x$ relative to the measure $\pi$. The question of almost sure convergence is open.

In the case of continuous time Markov processes we have the transition probabilities $p(t, x, d y)$ and an invariant probability measure $\pi(d x)$ with respect to which the process is assumed to be reversible and ergodic. We also have the infinitesimal generator $L$ of the process. $L$ is self adjoint on $L_{2}(\pi)$ with a nonpositive spectrum and a simple eigenvalue of 0 , constants being the eigenspace corresponding to that eigenvalue.

If one is interested in proving a central limit theorem for an additive functional of the form

$$
X(t)=\int_{0}^{t} V(y(s)) d s
$$

where $\int V d \pi=0$, then

$$
\begin{aligned}
\lim _{t \rightarrow \infty} \frac{1}{t} E^{P}\left[X^{2}(t)\right] & =2 \int_{0}^{\infty} E^{P}[V(y(s)) V(y(0))] d s=2 \int_{0}^{\infty}\left\langle T_{s} V, V\right\rangle d s \\
& =2\left\langle-L^{-1} V, V\right\rangle,
\end{aligned}
$$

where $T_{s}$ is the semigroup induced by the transition probabilities.

The natural assumption on $V$ then is that $V \in D\left((-L)^{-1 / 2}\right)$ or equivalently an estimate of the form

$$
|\langle V, \phi\rangle| \leqq c\langle-L \phi, \phi\rangle^{1 / 2}
$$

for all $\phi$ in the domain of $L$. We will assume that $V$ in $L_{2}(\pi)$ satisfies (1.14). When we say domain of $L$ we mean the domain as the generator of a semigroup in $L_{2}(\pi)$.

We now state the analog of Theorem 1.3.

Theorem 1.8. Let $y(t)$ be a Markov process, reversible with respect to a probability measure $\pi$, and let us suppose that the reversible stationary process $P$ with $\pi$ as invariant measure is ergodic. Let $V$ be a function on the state space in $L_{2}(\pi)$ satisfying $\int$ $V d \pi=0$ and condition (1.14). Let $F_{t}$ be the $\sigma$-field generated by the process up to time $t$. There exists a square integrable Martingale $M_{t}$ relative to $\left(\Omega, F_{t}, P\right)$ for $f \geqq 0$ such that $M_{t}$ has stationary increments and

$$
\lim _{t \rightarrow \infty} \frac{1}{\sqrt{ } t} \sup _{0 \leqq s \leqq t}\left|x(s)-M_{s}\right|=0
$$

in probability with respect to $P$, where $X(0)=M_{0}=0$ and

$$
X(t)=\int_{0}^{t} V(y(s)) d s
$$


Moreover

$$
\lim _{t \rightarrow \infty} \frac{1}{t} E^{P}\left|X(t)-M_{t}\right|^{2}=0
$$

Corollary 1.9. It follows now from results is Helland [2] that $1 / \sqrt{ } \lambda X(\lambda t)$ satisfies a functional central limit theorem relative to $P$ and the limiting variance $\sigma^{2}$ is given by

$$
\sigma^{2}=\lim _{t \rightarrow \infty} \frac{1}{t} E X^{2}(t)=2 \int_{0}^{\infty}\left\langle T_{t} V, V\right\rangle d t=2\left\langle-L^{-1} V, V\right\rangle .
$$

Remark 1.10. If we denote by $P_{x}$ the Markov process starting from a point $x$ in the state space and by $Q_{\lambda, x}$ the measure on the space of continuous functions induced by $1 / \sqrt{ } \lambda X(\lambda t)$ from $P_{x}$ and by $Q$ the Brownian motion measure with variance $\sigma^{2}$ on the same function space, then we have besides $\int Q_{\lambda, x} \pi(d x) \Rightarrow Q$ actually $Q_{\lambda, x} \Rightarrow Q$ in measure with respect to $\pi$. The question of almost sure convergence is open.

Remark 1.11. If $V$ is a vector of functions one has analogous results regarding convergence to the corresponding vector Brownian motion with appropriate covariance matrix. The basic approximation in Theorem 1.8 can be done for each component and in [2] the multidimensional version of the central limit theorem for vector martingales can be found.

Comments on Proofs. The proof follows the discrete time situation very closely. The only tricky point is the analog of Lemma 1.4. Since the $X(t)$ process is almost surely continuous and $M_{t}$ can be taken to be almost surely right continuous, one replaces the supremum in

$$
\sup _{0 \leqq s \leqq t}\left|X(s)-M_{s}\right|
$$

by a supremum over diadic points in $[0, t]$. Denoting by $D_{N}$ the set of diadic points of the form $j / 2^{N}$, it is clearly sufficient to prove

Lemma 1.12. Let $f$ satisfy $\langle f, f\rangle=a,\langle f,-L f\rangle=b$. Then for every $t, N$ and $l$

$$
P\left[\sup _{0 \leqq s \leqq t, s \in D_{N}} \mid f(y(s) \mid \geqq l] \leqq \frac{3}{l} \sqrt{a+t b} .\right.
$$

Proof. If we denote by $q_{N}(x, d y)$ the transition probability $p\left(1 / 2^{N}, x, d y\right)$ and by $q_{N}$ the operator $T_{1 / 2^{N}}$, then by the spectral theorem,

$$
\left\langle f,\left(I-q_{N}\right) f\right\rangle=\int\left(1-e^{-\lambda / 2^{N}}\right) E_{f}(d y) \leqq \frac{1}{2^{N}}\langle f,-L f\rangle=\frac{b}{2^{N}} .
$$

We now apply Lemma 1.4 for the discretely sampled process with $n=\left[t 2^{N}\right]$, and we obtain the validity of the lemma.

\section{Asymptotics for a Single Test Particle in Simple Exclusion}

In this section we study the behavior of one test particle in the symmetric simple exclusion process. We will denote by $\xi \in\{0,1\}^{\mathbb{Z}^{d}}$ the state of such a process 


$$
\xi(u)= \begin{cases}1 & \text { if the site } u \text { has a particle } \\ 0 & \text { if the site } u \text { is free. }\end{cases}
$$

For any state $\xi$ and any two sites $u, v$ we denote by $\xi^{u, v}$ the new state obtained by

$$
\xi^{u, v}(z)=\begin{array}{lll}
\xi(z) & \text { if } & z \neq u \text { or } v \\
\xi(u) & \text { if } & z=v \\
\xi(v) & \text { if } & z=u .
\end{array}
$$

The evolution of the process $\xi(t, \cdot)$ is governed by its infinitesimal generator $L$

$$
(L F)=\sum_{u \neq v}\left[F\left(\xi^{u, v}\right)-F(\xi)\right] p(u-v)
$$

The summation here is over unordered pairs $(u, v)$ with $u \neq v$. For the process $\xi(t, \cdot)$ the Bernoulli measures $P_{\theta}$, where $\theta$ is the probability that any site is occupied (independently of all the other sites) are reversible ergodic measures provided the random walk $p(x-y)$ is irreducible.

If we have a single test particle in the system, it is more convenient to describe the system in terms of the location $x$ of the test particle and the configuration $\eta$ of the entire system seen from the test particle:

$$
\eta(u)=\xi(x+u) \text { for } u \neq 0,
$$

and the state space for the system is $x \in \mathbb{Z}^{d}$ and $\eta \in\{0,1\} \mathbb{Z}^{d}-\{0\}$. The infinitesimal generator for the $(x, \eta)_{t}$ process is given by

$$
\begin{aligned}
(\tilde{L} F)(x, \eta)= & \sum_{z \neq 0} p(z)(1-\eta(z))\left[F\left(x+z, \tau_{-z} \eta\right)-F(x, \eta)\right] \\
& +\sum_{u \neq v \neq 0}\left[F\left(x, \eta^{u, v}\right)-F(x, \eta)\right] p(u-v),
\end{aligned}
$$

where

$$
\begin{aligned}
\left(\tau_{-z} \eta\right)(u) & =\eta(u+z) \text { for } u \neq-z \\
& =0 \text { for } u=-z .
\end{aligned}
$$

From the form of (2.3) we see that $\eta$ by itself is a Markov process with generator

$$
\begin{aligned}
\left(L_{0} F\right)(\eta) & =\sum_{z} p(z)(1-\eta(z))\left[F\left(\tau_{-z} \eta\right)-F(\eta)\right]+\sum_{u \neq v \neq 0}\left[F\left(\eta^{u, v}\right)-F(\eta)\right] p(u-v) \\
& =\left(L_{1} F\right)(\eta)+\left(L_{2} F\right)(\eta) .
\end{aligned}
$$

The invariant measures for the $\eta$ process will turn out to be Bernoulli product measures $P_{\theta}$ with common probability $\theta$, and these will be ergodic and reversible for $L_{0}$. [Although for the $\eta$ process there is no site at 0 we will continue to denote by $\boldsymbol{P}_{\boldsymbol{\theta}}$ the Bernoulli measure over all the other sites.]

Lemma 2.1. The $\eta_{t}$ process is reversible and ergodic with respect to $\boldsymbol{P}_{\boldsymbol{\theta}}$.

Proof. We need only check that

$$
\int f L_{0} g d P_{\theta}=\int g L_{0} f d P_{\theta},
$$


where $f$ and $g$ are of the form

$$
f(\eta)=\prod_{u \in A} \eta(u), \quad g(\eta)=\prod_{u \in B} \eta(u)
$$

where $A, B \subset \mathbb{Z}^{d}-\{0\}$.

From the form of the generator (2.5) we need only check that

$$
\begin{gathered}
\int f\left(\eta^{u, v}\right) g(\eta) d P_{\theta}=\int g\left(\eta^{u, v}\right) f(\eta) d P_{\theta}, \\
\int f(\eta) g\left(\tau_{-z} \eta\right)(1-\eta(z)) d P_{\theta}=\int g(\eta) f\left(\tau_{z} \eta\right)(1-\eta(-z)) d P_{\theta} .
\end{gathered}
$$

Equation (2.6) follows from the invariance of $P_{\theta}$ under the map $\eta \rightarrow \eta^{u, v}$ and (2.7) follows from the fact that $\eta \rightarrow \tau_{z} \eta$ maps $(1-\eta(z)) d P_{\theta}$ to $(1-\eta(-z)) d P_{\theta}$. It is easy to see that $P_{\theta}$ is reversible and ergodic for $L_{2}$. Since any harmonic function $\phi$ such that $\left(L_{1}+L_{2}\right) \phi=0$ has the property $\int \phi L_{1} \phi d P_{\theta}=-\int \phi L_{2} \phi d P_{\theta}$ and $\int \phi L_{i} \phi d P_{\theta} \leqq 0$ for $i=1,2$ we have $\int \phi L_{1} \phi d P_{\theta}=\int \phi L_{2} \phi d P_{\theta}=0$. But $L_{2}$ is reversible and ergodic. Therefore $\phi$ is a constant almost surely with respect to $P_{\theta}$. This completes the proof of the lemma. then

The $x_{t}$ process is driven by the $\eta_{t}$ process. If we pick a vector $l$ and consider $(x \cdot l)$,

$$
L_{0}(x \cdot l)=\sum(z \cdot l) p(z)(1-\eta(z))=\phi_{l}(\eta)
$$

so that

$$
\left(x_{t} \cdot l\right)-\int_{0}^{t} \phi_{l}\left(\eta_{s}\right) d s=N_{t}^{l}
$$

is a Martingale relative to the natural $\sigma$-fields. We now apply Theorem 1.3 to $\phi_{l}(\eta)$ and write

$$
\int_{0}^{t} \phi_{l}\left(\eta_{s}\right) d s=M_{t}^{l}+E_{t}^{l}
$$

where $M_{t}^{l}$ is another martingale and $E_{t}^{l}$ is negligible. Taking $l$ to be the various basis vectors, we can approximate each component of $x_{t}$ by a sum of two martingales. The central limit theorem now follows from what we said earlier. We need to show that the conditions of Theorem 1.3 apply and that the limiting covariance of the sum $M_{t}+N_{t}$ is nondegenerate. We assume that

$$
\sum|z|^{2} p(z)<\infty
$$

Lemma 2.2. For any $l$ the function

$$
\phi_{l}(\eta)=\sum(z \cdot l)(1-\eta(z)) p(z)
$$

satisfies the condition

$$
\left|\int \phi_{l}(\eta) F(\eta) d P_{\theta}\right| \leqq C\left(-\int\left(L_{0} F\right)(\eta) F(\eta) d P_{\theta}\right)^{1 / 2}
$$

Proof. We can take $F$ to be a function depending on a finite number of coordinates and then 


$$
\begin{aligned}
\int \phi_{l}(\eta) F(\eta) d P_{\theta} & =\int\left(\sum(z, l)(1-\eta(z)) p(z)\right) F(\eta) d P_{\theta} \\
& =\frac{1}{2} \int\left(\sum_{z}(z \cdot l)[(1-\eta(z))-(1-\eta(-z))] F(\eta) p(z)\right) d P_{\theta} \\
& =\frac{1}{2} \int \sum(z \cdot l)(1-\eta(z))\left[F\left(\tau_{-z} \eta\right)-F(\eta)\right] p(z) d P_{\theta} \\
& \leqq \frac{1}{2}\left(\sum p(z)(z \cdot l)^{2}\right)^{1 / 2}\left(\int \sum(1-\eta(z))\left[F\left(\tau_{-z} \eta\right)-F(\eta)\right]^{2} p(z) d P_{\theta}\right)^{1 / 2} \\
& \leqq C\left(\int\left(-L_{1} F\right)(\eta) F(\eta) d P_{\theta}\right)^{1 / 2} \\
& \leqq C\left(-\int\left(L_{0} F\right)(\eta) F(\eta) d P_{\theta}\right)^{1 / 2}
\end{aligned}
$$

For the next lemma which is required to prove the nondegeneracy of the limiting covariance we will assume that under the random walk $p(u-v)$ one can get from any nonzero site to any other nonzero site without going through the origin. Notice that this rules out only the nearest neighbor random walk in one dimension.

Lemma 2.3. Under the above assumption we have an estimate of the form

$$
\left|\int \phi_{l}(\eta) F(\eta) d P_{\theta}\right| \leqq C\left(-\int\left(L_{2} F\right)(\eta) F(\eta) d P_{\theta}\right)^{1 / 2}
$$

Proof. It is clearly sufficient to prove (see (2.9))

$$
\left|\int[(1-\eta(u))-(1-\eta(v))] F(\eta) d P_{\theta}\right| \leqq C|u-v|\left(-\int\left(L_{2} F\right)(\eta) F(\eta) d P_{\theta}\right)^{1 / 2} .
$$

In view of the triangle inequality is to enough to establish

$$
\sup _{|u-v| \leqq k}\left|\int[(1-\eta(u))-(1-\eta(v))] F(\eta) d P_{\theta}\right| \leqq C\left(-\int\left(L_{2} F\right)(\eta) F(\eta) d P_{\theta}\right)^{1 / 2}
$$

The estimate is going to be derived through a random walk path from $u$ to $v$ that avoids zero. Since for a fixed path of finite length, translations will produce only at most a finite number that will go through zero the supremum in (2.10) comes for free provided we establish (2.10) for each fixed $u$ and $v$ (with "translation invariant" $C$, see below). Let

$$
u=z_{0}, \quad z_{1}, \ldots z_{n}=v
$$

be a path that avoids 0 , and let $p\left(z_{i}-z_{i-1}\right)>0$ for $i=1,2, \ldots, n$. Again because of the triangle inequality we can concentrate on

$$
\begin{aligned}
& \left|\int\left[\left(1-\eta\left(z_{i}\right)\right)-\left(1-\eta\left(z_{i-1}\right)\right)\right] F(\eta) d P_{\theta}\right|=\left|\int\left(1-\eta\left(z_{i-1}\right)\right)\left(F\left(\eta^{z_{i-1}, z_{i}}\right)-F(\eta)\right) d P_{\theta}\right| \\
& \leqq\left(\int\left[F\left(\eta^{z_{i-1}, z_{i}}\right)-F(\eta)\right]^{2} d P_{\theta}\right)^{1 / 2} \\
& \leqq\left(\frac{1}{p\left(z_{i}-z_{i-1}\right)}\right)^{1 / 2}\left(-\int\left(L_{2} F\right)(\eta) F(\eta) d P_{\theta}\right)^{1 / 2}
\end{aligned}
$$

We are finally ready to state and prove our main theorem: Let us consider a test particle in simple exclusion where the underlying random walk probabilities $p(u-v)$ are assumed to be symmetric and satisfying $\sum|z|^{2} p(z)<\infty$. Moreover the random walk is assumed to be irreducible and not the one dimensional nearest neighbor random walk. The test particle is assumed to start at 0 . The rest of the configuration is distributed according to the invariant Bernoulli product measure $P_{\theta}$. Then 
Theorem 2.4. The distribution of $(1 / \sqrt{ } \lambda) x_{\lambda t}$ converges to a nondegenerate diffusion with covariance $\sigma$ in the Skorohod space. Moreover the covariance $\sigma$ is also the limit of the covariances of $(1 / \sqrt{ } \lambda) x_{\lambda}$ as $\lambda \rightarrow \infty$.

Proof. In view of the earlier comments we need only prove nondegeneracy. There are many martingales associated with the $(x, \eta)_{t}$ process;

$$
\begin{aligned}
v_{t}^{u, v} & =\sum_{s \leqq t} \chi_{\left(\eta_{s_{-}}=\eta_{s}^{u, v}\right)}\left|\eta_{s}(u)-\eta_{s}(v)\right|-\int_{0}^{t}\left|\eta_{s}(u)-\eta_{s}(v)\right|-\int_{0}^{t}\left|\eta_{s}(u)-\eta_{s}(v)\right| p(u-v) d s \\
v_{t}^{z} & =\sum_{s \leqq t} \chi_{\left(\eta_{s}=\tau_{-z} \eta_{s_{-}}\right)}-\int_{0}^{t} p(z)\left(1-\eta_{s}(z)\right) d s
\end{aligned}
$$

are basic martingales, and any other martingale is expressible in terms of these. For instance $N_{t}$ given by (2.7) can be expressed as

$$
N_{t}=\sum_{z \neq 0} z v_{t}^{z}
$$

For any function $F$ the martingale

$$
M_{t}^{F}=F\left(\eta(t)-F(\eta(0))-\int_{0}^{t}\left(L_{0} F\right)(\eta(s)) d s\right.
$$

can be expressed as

$$
M_{t}^{F}=\int_{u, v \neq 0} \int_{0}^{t}\left[F\left(\eta^{u, v}(s)\right)-F(\eta(s))\right] d v_{s}^{u, v}+\sum_{z} \int_{0}^{t}\left[F\left(\tau_{-z} \eta(s)\right)-F(\eta(s))\right] d v_{s}^{z} .
$$

Moreover the martingales $\left\{v_{t}^{z} v_{t}^{u, v}\right\}$ are mutually orthogonal in the sense that the product of any two distinct ones is again a martingale. For any $l$ the martingale $\left\langle N_{t}, l\right\rangle$ is of the form

$$
\left\langle N_{t}, l\right\rangle=\sum_{z \doteqdot 0}(z, l) v_{t}^{z}
$$

and $\left\langle M_{t}, l\right\rangle$ of the decomposition

$$
\int_{0}^{t} \phi_{l}\left(\eta_{s}\right) d s=M_{t}^{l}+E_{t}^{l}
$$

comes from $M_{t}^{F_{\lambda}}$ as $\lambda \rightarrow 0$, where $F_{\lambda}=\left(\lambda I-L_{0}\right)^{-1} \phi_{l}$. From the orthogonality of these martingales it is clear that the nondegeneracy of $M_{t}+N_{t}$ is assured if we establish that $M_{t}$ should involve at least some $v_{t}^{u, v}$ component so that it cannot cancel $N_{t}$. In other words we need to prove

$$
\lim _{\lambda \rightarrow 0} E^{P_{\theta}}\left\{\sum_{u \neq v \neq 0} p(u-v)\left[F_{\lambda}\left(\eta^{u, v}(s)\right)-F_{\lambda}(\eta(s)]^{2}\right\} \neq 0\right.
$$

i.e.

$$
\lim _{\lambda \rightarrow 0}\left\langle-L_{2}\left(\lambda I-L_{0}\right)^{-1} \phi_{l}, \quad\left(\lambda I-L_{0}\right)^{-1} \phi_{l}>\neq 0 .\right.
$$


Lemma 2.3 assures us that

or

$$
\left|\left\langle\phi_{l}, \psi\right\rangle\right| \leqq C\left\langle-L_{2} \psi, \psi\right\rangle^{1 / 2}
$$

$$
\left\langle-L_{2}^{-1} \phi_{l}, \phi_{l}\right\rangle \leqq C \text {. }
$$

We can estimate

$$
\begin{aligned}
\left\langle\phi_{l},\left(\lambda I-L_{0}\right)^{-1} \phi_{l}\right\rangle & =\left\langle\left(-L_{2}^{-1 / 2} \phi_{l},\left(-L_{2}\right)^{1 / 2}\left(\lambda I-L_{0}\right)^{-1} \phi_{l}\right\rangle\right. \\
& \leqq\left\langle-L_{2}^{-1} \phi_{l}, \phi_{l}\right\rangle^{1 / 2}\left\langle-L_{2}\left(\lambda I-L_{0}\right)^{-1} \phi_{l},\left(\lambda I-L_{0}\right)^{-1} \phi_{l}\right\rangle \\
& \leqq C\left\langle-L_{2}\left(\lambda I-L_{0}\right)^{-1} \phi_{l},\left(\lambda I-L_{0}\right)^{-1} \phi_{l}\right\rangle .
\end{aligned}
$$

Since $\left\langle\phi_{l},\left(\lambda I-L_{0}\right)^{-1} \phi_{l}\right\rangle$ can go to zero only if $\phi_{l}=0$, we are done.

Corollary 2.5. Suppose the initial configuration in the sites $u \neq 0$ is different from $P_{\theta}$ but is some measure $Q \ll P_{\theta}$. Then the basic central limit theorem is still valid and the limiting distribution is the same as before. If $d Q / d P_{\theta}$ is bounded, then the covariance of $(1 / \sqrt{ } \lambda) x_{\lambda}$ converges to the covariance of the limiting Brownian motion. The proof follows easily from Remark 1.10 . The boundedness of $d Q / d P_{\theta}$ guarantees that the error is still negligible in the sense of mean square.

\section{Asymptotic Independence of Two Test Particles}

In this section we study the behavior of two test particles located initially at points $x$ and $y$.

Theorem 3.1. The scaled positions of two test particles converge jointly to two independent Brownian motions.

Proof. First of all we note that we are working with an initial distribution conditioned to having sites $x$ and $y$ occupied. Since the conditioning set has nonzero measure by Corollary 2.5, the two individual positions converge to Brownian motions separately and only asymptotic independence has to be proved. In view of the results in Helland [2] in order to prove that two Martingales satisfying the central limit theorem separately converge jointly, after rescaling, to independent Brownian motions we need only show that

$$
\lim _{t \rightarrow \infty} \frac{1}{t} E M_{i, t}^{(1)} M_{j, t}^{(2)}=0
$$

where $M_{i, t}^{(j)}$ is the $i^{\text {th }}$ component of the $j^{\text {th }}$ martingale. By standard formula one can compute

$$
E M_{1 t}^{(1)} M_{j t}^{(2)}=E \int_{0}^{t} \psi_{i j}(s) d s
$$

for some suitable function $\psi_{i j}(s)$.

We will carry out these computations in the next several lemmas and establish that $E \psi_{i j}(s) \rightarrow 0$ as $s \rightarrow \infty$. This will prove the result.

First some notation. The probability that a typical site is occupied is of course $\theta$ and is fixed. We will not specify it hereafter. We have the initial measures $P, P_{x}, P_{y}$, 
$P_{x y}$ corresponding to no conditions, conditioned for site $x$, site $y$ and both sites $x$ and $y$ to be occupied respectively at time 0 . We also have the measures for the entire process $\xi_{t}$ of the infinite particle system corresponding to these initial conditions which we denote by $Q, Q_{x}, Q_{y}$ and $Q_{x y}$ respectively.

Lemma 3.2. The positions $x_{t}$ and $y_{t}$ of two tagged particles starting from $x$ and $y$ at time $t=0$ can be represented as

$$
x_{t}=x+M_{t}^{(1)}+E_{t}^{(1)}, \quad y_{t}=y+M_{t}^{(2)}+E_{t}^{(2)},
$$

where $M_{t}^{(1)}$ and $M_{t}^{(2)}$ are martingales relative to $Q_{x}$ and $Q_{y}$ respectively. They are both martingales relative to $Q_{x, y}$. Further $E_{t}^{(1)}, E_{t}^{(2)}$ are negligible relative to $Q_{x, y}$ in the mean square sense.

Proof. The representation of $x_{t}$ relative to $Q_{x}$ and $y_{t}$ relative to $Q_{y}$ follow from Sect. 2 . Since all martingale properties are valid relative to almost all initial starting points and $P_{x, y} \ll P_{x}$ as well as $P_{x, y} \ll P_{y}$, the martingale property holds true for $Q_{x, y}$ as well. Moreover $d Q_{x, y} / d Q_{x}$ and $d Q_{x, y} / d Q_{y}$ are bounded. So all the negligibility results are valid in the mean square sense as well.

Let us look at the complete class of martingales for the $\xi_{t}$ process for any $a, b \in \mathbb{Z}^{d}$ and $a \neq b$ :

$$
n(t, a, b)=N_{t}(a, b)-\int_{0}^{t} p(b-a) \xi_{s}(a)\left(1-\xi_{s}(b)\right) d s
$$

is a martingale where $N_{t}(a, b)$ is the number of transitions in time $[0, t]$ from site $a$ to site $b$. Since any martingale is expressible in terms of these basic ones

$$
M_{i, t}^{(1)}=\sum_{a \neq b} \int_{0}^{t} \phi_{i, a, b}^{(1)}(s) d n(s, a, b)
$$

and

$$
M_{i, t}^{(2)}=\sum_{a \neq b} \int_{0}^{t} \phi_{i, a, b}^{(2)}(s) d n(s, a, b) .
$$

Lemma 3.3. For each $t>0$

$$
E^{Q_{x, y}} M_{i, t}^{(1)} M_{j, t}^{(2)}=E^{Q_{x, y}} \int_{0}^{t} \psi_{i j}(s) d s
$$

where

$$
\psi_{i j}(s)=\sum_{a \neq b} \phi_{i, a, b}^{(1)}(s) \phi_{j, a, b}^{(2)}(s) \eta_{s}(a)\left(1-\eta_{s}(b)\right) p(b-a) .
$$

Proof. Follows from the fact that unless $a=a^{\prime}$ and $b=b^{\prime}$ the martingales $n(t, a, b)$ and $n\left(t, a^{\prime}, b^{\prime}\right)$ are orthogonal and

$$
E n^{2}(t, a, b)-\int_{0}^{t} \eta_{s}(a)\left(1-\eta_{s}(b)\right) p(b-a) d s
$$

is a martingale. 
Lemma 3.4. For any $L<\infty$,

$$
\lim _{t \rightarrow \infty} Q_{x, y}[|x(t)-y(t)| \leqq L]=0 .
$$

Proof. Let us consider the system consisting of the first tagged particle being the origin of reference and the rest of the world including the second tagged particle being viewed from the first tagged particle. For this system there is a $\sigma$-finite invariant measure with mass one corresponding to each location of the second tagged particle. Given the location of the second tagged particle the rest of the universe (excluding also the origin) is provided a Bernoulli product measure. One checks that except for the one dimensional nearest neighbor model the system is ergodic. Moreover the system is reversible so that if $T_{t}$ is the evolution semigroup for the system $\left\langle f, T_{t} g\right\rangle \rightarrow 0$ by the spectral theorem provided $f$ and $g$ are square integrable with respect to the invariant measure. Picking $g=\chi_{\{|u| \leqq L\}}(\cdot)$ and $f=\chi_{y}$, i.e. the indicators of the sets where the second tagged particle is within a distance $L$ of the origin and at $y$ respectively we obtain our result.

\section{Lemma 3.5.}

$$
\lim _{t \rightarrow \infty} E^{Q_{x, y}} \psi_{i j}(t)=0
$$

Proof. We use the simple inequality

$$
\begin{aligned}
\left|\psi_{i j}(t)\right| \leqq & \chi E_{1}(t) \cap E_{2}(t) \neq \phi \cdot\left[Q_{i}^{(1)}(t)\right]^{1 / 2}\left[Q_{j}^{(2)}(t)\right]^{1 / 2} \\
& +\left[Q_{i}^{(1)}(t)\right]^{1 / 2}\left[Q_{j, E_{2}^{t}(t)}^{(2)}(t)\right]^{1 / 2}+\left[Q_{i, E_{1}^{c}(t)}^{(1)}(t)\right]^{1 / 2}\left[Q_{j}^{(2)}(t)\right]^{1 / 2}
\end{aligned}
$$

where $L<\infty$ is fixed, and

$$
\begin{aligned}
E_{1}(t) & =\left\{(u, v) \in \mathbb{Z}^{d} \times \mathbb{Z}^{d}, u \neq v \text { and } \max \left[\left|u-x_{t}\right|,\left|v-x_{t}\right|\right] \leqq L\right\}, \\
E_{2}(t) & =\left\{(u, v) \in \mathbb{Z}^{d} \times \mathbb{Z}^{d}, u \neq v \text { and } \max \left[\left|u-y_{t}\right|,\left|v-y_{t}\right|\right] \leqq L\right\}, \\
Q_{i}^{(r)}(t) & =\sum_{u, v}\left|\phi_{i, u, v}^{(r)}(t)\right|^{2} \eta_{t}(u)\left(1-\eta_{t}(v)\right) p(v-u), \\
Q_{i, A}^{(r)}(t) & \sum_{(u, v) \in A}\left|\phi_{i, u, v}^{(r)}(t)\right|^{2} \eta_{t}(u)\left(1-\eta_{t}(v)\right) p(v-u) .
\end{aligned}
$$

We write for each $L<\infty$

$$
E^{Q_{x, y}}\left|\psi_{i j}(t)\right| \leqq a_{L}(t)+b_{L}(t)+c_{L}(t)
$$

corresponding to the estimate (3.3), which we think of as

$$
\psi_{i j}(t) \leqq T_{1, L}(t)+T_{2, L}(t)+T_{3, L}(t) .
$$

Step 1.

$$
\begin{aligned}
a_{L}(t)=E^{Q_{x, y}} T_{1, L}(t) & \leqq \int_{\left|x_{t}-y_{t}\right| \leqq 2 L}\left[Q_{i}^{(1)}(t) Q_{j}^{(2)}(t)\right]^{1 / 2} d Q_{x, y} \\
& \leqq\left(\int_{\left|x_{t}-y_{t}\right| \leqq 2 L} Q_{i}^{(1)}(t) d Q_{x, y}\right)^{1 / 2}\left(\int_{\left|x_{t}-y_{t}\right| \leqq 2 L} Q_{j}^{(2)}(t) d Q_{x, y}\right)^{1 / 2}
\end{aligned}
$$


To show that $a_{L}(t) \rightarrow 0$ as $t \rightarrow 0$, it suffices to know that $Q_{i}^{(\mathrm{r})}(t)$ are uniformly integrable with respect to $Q_{x, y}$. But $d Q_{x, y} / d Q_{x}$ and $d Q_{x, y} / d Q_{y}$ are bounded and $Q_{i}^{(1)}(t)$ and $Q_{i}^{(2)}(t)$ are stationary processes relative to $Q_{x}$ and $Q_{y}$ respectively.

Step 2.

$$
\begin{aligned}
b_{L}(t)=E^{Q_{x, y}} T_{2, L}(t) & \leqq\left(E^{Q_{x, y}}\left[Q_{i}^{(1)}(t)\right]\right)^{1 / 2}\left(E^{Q_{x, y}}\left[Q_{j, E_{2}(t)}^{(2)}(t)\right]\right)^{1 / 2} \\
& \leqq C\left(E^{Q_{x}}\left[Q_{i}^{(1)}(t)\right]\right)^{1 / 2}\left(E^{Q_{y}}\left[Q_{j, E_{2}^{(}(t)}^{(2)}(t)\right]\right) .
\end{aligned}
$$

$E^{Q_{x}}\left[Q_{i}^{(1)}(t)\right]$ is independent of $t$ and so is $E^{Q_{y}}\left[Q_{j, E_{2}^{c}(t)}^{(2)}(t)\right]$. Moreover the last quantity tends to zero as $L \rightarrow \infty$, and we are done.

Step 3. The estimate for $c_{L}(t)$ is identical to step 2 with the roles of the two tagged particles reversed.

\section{Application to a Bond Diffusion Process}

In this section we want to study the movement of a particle in a random environment. Consider for each bond $b$ of $Z^{d}$ a positive random variable $a_{b}$ and suppose that these random variables are i.i.d.. Once this random environment is chosen, let a particle evolve according to the jump process with generator $L f(x)=\sum_{y=x} a_{y x}[f(y)-f(x)]$, where $y \sim x$ means that $y$ and $x$ are nearest-neighbors and $a_{y x}$ is the rate of the corresponding bond. We also assume that the $a_{b}$ 's have a uniform upper and lower bound. Call $x_{t}$ the position of the particle at time $t$, and $\varepsilon x_{t / \varepsilon^{2}}$ the rescaled position. Then

Theorem 4.1. After rescaling the position of the particle converges to Brownian motion.

Proof. The only quantities that matter in this problem are the values of the rates given to each bond (which we call an environment).

It is also clear that the process $\xi_{t}$ of the environment seen from the position of the particle at time $t$ is a Markov process whose generator is

$$
\widetilde{L} f(\xi)=\sum_{e} a_{e}(\xi)\left[f\left(\tau_{e} \xi\right)-f(\xi)\right]
$$

Therefore to prove our central limit theorem we only have to check that, for any $\vec{\alpha} \in R^{d}$, the function

$$
\phi(\xi)=\sum a_{e}(\xi)(\vec{e} \cdot \vec{\alpha})
$$

satisfies the condition of Theorem 1.3, because we easily check that the original product measure (say $m(d \xi)$ ) is reversible for this process;

$$
\begin{aligned}
\int a_{e}(\xi)(e \cdot \alpha) g(\xi) m(d \xi) & =\int a_{-e}\left(\tau_{e} \xi\right)(\vec{e} \cdot \vec{\alpha}) g(\xi) m(d \xi) \\
& =-\int a_{-e}(\eta)(-\vec{e} \cdot \vec{\alpha}) g\left(\tau_{-e} \eta\right) m(d \eta)
\end{aligned}
$$

Therefore

$$
\sum_{e} \int g(\xi) a_{e}(\xi)(\vec{e} \cdot \vec{\alpha}) m(d \xi)=\frac{1}{2} \int \sum_{e} a_{e}(\xi)(\vec{e} \cdot \vec{\alpha})\left(f\left(\tau_{e} \xi\right)-f(\xi)\right) m(d \xi)
$$




$$
\leqq \frac{1}{2}\left[\sum_{e} \int_{e} a_{e}(\xi)(\vec{e} \cdot \vec{\alpha})^{2} m(d \xi)\right]\left[\sum_{e} \int a_{e}(\xi)\left(f\left(\tau_{e} \xi\right)-f(\xi)\right)^{2} m(d \xi)\right]
$$

which is the bound that we were looking for.

Remark 4.2. With some additional work one can prove the nondegeneracy of the limiting Brownian motion.

Acknowledgements. C. K. acknowledges the warm hospitality of the CIMS where this work was done. The authors wish to thank S. Goldstein and H. Spohn for valuable discussions as well as detailed comments on an earlier version of the paper which are responsible for this improved version.

\section{References}

1. Arratia, R.: The motion of a tagged particle in the simple symmetric exclusion system in Z. Ann. Probab. 11 362-373 (1983)

2. Helland, I.: Central limit theorems for martingales with discrete or continuous time. Scand. J. Stat., 9, 79-94 (1982)

3. Kipnis, C., Lebowitz, J. L., Presutti, E., Spohn, H.: J. Stat. Phys. 30, 107-121 (1983)

4. Lebowitz, J. L., Spohn, H.: J. Stat. Phys. 28, 539-556 (1982)

Communicated by J. L. Lebowitz

Received August 10, 1984; in revised form July 2, 1985 
